\title{
BRCA1 and BRCA2 mutation analysis in 86 early onset breast/ovarian cancer patients
}

\author{
Alex M Garvin, Michele Attenhofer-Haner, Rodney J Scott
}

\begin{abstract}
Eighty-six women fulfilling specific selection criteria were studied for germline mutations in two breast cancer susceptibility genes, BRCA1 and BRCA2, using the protein truncation test (PTT). Nine germline mutations were identified, six in BRCA1 and three in BRCA2. Of the six BRCA1 mutations, three have previously been described and three are new, and for BRCA2, one is a new mutation and the other two appear to occur at a site that has been described several times. Four kindreds were breast cancer families, one a breast/ovarian cancer family, and the sixth an ovarian cancer family. The three kindreds with BRCA2 mutations were classified as one breast/ovarian cancer family, one breast cancer family, and one family which harboured one early onset breast cancer patient and two melanoma patients. The mutations in BRCA1 were either insertions, deletions, or transitions which all resulted in a premature stop codon. Mutations in BRCA2 were all frameshift mutations as a result of either 2 or 4 bp deletions. Two BRCA2 mutations were identical, suggesting a Swiss founder effect which was confirmed by haplotype sharing. The $10 \%$ mutation detection rate is compatible with the relaxed criteria used for patient selection. Considering the relative ease with which coding sequences can be screened by PTT, this assay is useful as a first screen for BRCA1 and BRCA2 mutations.

$(\mathcal{M}$ Med Genet 1997;34:990-995)
\end{abstract}

Keywords: breast cancer; BRCA1; BRCA2; genetics

The most consistent factor associated with a woman's risk of developing breast cancer is a family history of disease. Hereditary breast cancer is characterised by early onset, an excess of bilateral disease, and in some families an over-representation of ovarian cancer. Epidemiological studies have pointed to the existence of several breast cancer susceptibility Received 28 May 1997 publication 23 July 1997

BRCA2

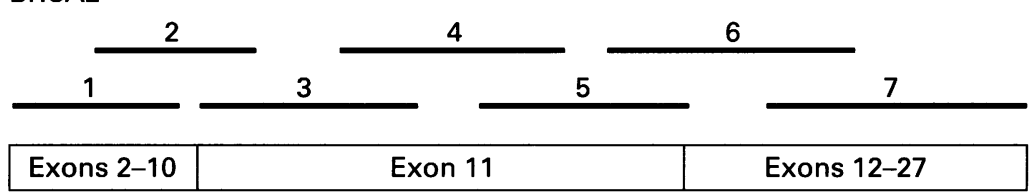

Figure 1 Diagrammatic representation of the coding sequence of BRCA2 indicating the seven overlapping segments that were amplified using the primer sequences indicated in table 1. genes, two of which have been recently identified, termed BRCA1 and BRCA2. ${ }^{12}$

The locus for BRCA1 was identified in 1990 and the gene identified by positional cloning four years later. ${ }^{1}{ }^{3}$ The BRCA2 locus was identified in 1994 and the gene identified in $1995 .{ }^{2}{ }^{4}$ BRCA1 confers a lifetime risk of approximately $85 \%$ of developing breast cancer and a $50 \%$ risk of developing ovarian cancer. ${ }^{5}$ BRCA2, however, confers a similar risk for breast cancer development but a different lifetime risk of developing ovarian cancer (only about $10 \%$ ) in comparison to BRCA1..$^{67}$ In addition, BRCA2 appears to be associated with an increased risk of male breast cancer whereas BRCA1 does not. ${ }^{6}$ It is currently not clear if germline mutations in BRCA2 confer an increased risk of cancers other than breast and ovary.

The BRCAl gene is located on chromosome $17 \mathrm{q} 21$ and codes for a $7.5 \mathrm{~kb}$ transcript which is spread across $100 \mathrm{~kb}$ of genomic DNA. The gene consists of 24 exons of which 22 are coding. The first 10 and last 13 exons are relatively small whereas exon 11 represents over $60 \%$ of the entire coding sequence. The gene codes for a 1863 amino acid zinc finger containing protein of unknown function, which has recently been shown to interact with an additional protein termed BARD1. Missense mutations in BRCA1 disrupt binding to BARD1, suggesting that interaction with BARD1 is important in regulating BRCA1 function. ${ }^{8}$ Eighty-six percent of BRCA1 mutations are either nonsense or frameshift mutations which result in prematurely truncated proteins that lead to a disruption of BRCA1 function. ${ }^{9}$

BRCA2 is located on chromosome $13 \mathrm{q} 12-13^{4}$ and codes for a $10.5 \mathrm{~kb}$ transcript. The gene consists of 27 exons and, like BRCA1, the first 10 and last 16 exons are relatively short whereas exon 11 represents approximately $50 \%$ of the coding sequence. The function of the gene remains elusive but the sequence does have some similarities to BRCA1. Similar to BRCA1, most BRCA2 mutations result in premature termination codons.

In this report we have investigated 86 early onset breast/ovarian cancer patients for mutations in BRCA1 and BRCA2 using the protein truncation test (PTT). We have identified six mutations in BRCA1 and three mutations in BRCA2. All families harbouring BRCA1 mutations presented with typical disease characteristics for this breast/ovarian cancer predisposition. Two of the three families harbouring BRCA2 mutations presented with early onset 
Table 1 BRCA2 primers

\begin{tabular}{|c|c|c|c|}
\hline Segment & $5^{\prime}$ & $3^{\prime}$ & Size (bp) \\
\hline 1 external & gccgggagaagcgtgagggg (101-120) & tggtggtggctggccagctt (1930-1911) & \\
\hline 1 internal & T7-cctattggatccaaagaga $(232-250)$ & tggtggtggctggccagctt $(1930-1911)$ & 1736 \\
\hline 2 external & gaagatagttttcattatg (1141-1160) & cctttctattagctacttggaaag (2922-2901) & \\
\hline 2 internal & T7-aaaaatctacaaaaagtaaga $(1180-1200)$ & gagaaaagttcttcagagtctgg $(2879-2857)$ & 1737 \\
\hline 3 & T7-ggtttattgcattcttctgtg (2137-2157) & ttctttaatctgagtgtttc (4374-4355) & 2275 \\
\hline 4 & T7-ccaagctacatattgcagaag (3625-3645) & ctcgttgttttccttaatta (5865-58460) & 2278 \\
\hline 5 & T7-tcaaaaagtatcttttgaaa (5116-5136) & cccactaagataaggggctc $(7070-7051)$ & 1192 \\
\hline 6 external & tctgtccaggtatcagatgc $(6181-6200)$ & ctcttttgttgggcctccac (8810-8791) & \\
\hline 6 internal & T7-accaggcaagtcttttccaaa (6247-6268) & agatgatgtcttctccatcc $(8736-8717)$ & 2527 \\
\hline 7 external & gaagattattttggtaagga (7921-7940) & cgctgaggtaaatttgaaac (10620-10600) & \\
\hline 7 internal & T7-ctcataccctccaatgatgg (7987-8006) & ggtttgaaattatattccag (10560-10541) & 2611 \\
\hline
\end{tabular}

The numbers in parentheses refer to the positions according to Tavtigian $e t$ al, ${ }^{22}$ Genbank accession number U43746.

breast cancer and one case of ovarian cancer. The proband of the third BRCA2 family presented with early onset breast cancer and had a family history of skin cancer rather than breast cancer.

\section{Patients and methods}

Patients were ascertained as they presented with disease at various clinics throughout the German and Italian speaking parts of Switzerland. At the time of diagnosis the patients were asked if they would be willing to participate in a genetic screening study of BRCAl and BRCA2 if they fulfilled any of the following minimum criteria: any woman under 40 years of age with breast cancer and no other affected first or second degree relatives; any women under 50 years of age with a first degree relative also under 50 years of age with breast cancer; any woman under 50 years of age with breast cancer and a first degree relative with ovarian cancer at any age; any woman with ovarian cancer with a first degree relative with ovarian cancer at any age; any women with bilateral breast cancer both of which were identified before the age of 60 years; and any women with breast and ovarian cancer. Disease status in probands and affected relatives was confirmed in the majority of patients by pathology reports and in those cases where these were unavailable by death certificates. All women entering into this study signed an informed consent declaration.

TEMPLATE PREPARATION

For genomic DNA, patient peripheral blood lymphocytes (PBLs) were prepared from $10 \mathrm{ml}$ EDTA blood and DNA was isolated using the salting out procedure. ${ }^{10}$ Total RNA was pre-

Table 2 Summary of breast and ovarian cancer patients in the families of the 86 breast cancer patients

\begin{tabular}{lll}
\hline Index & No of relatives & No of families \\
\hline Bilateral Brca $<60$ & 0 & 10 \\
Brca $<40$ & 0 & 24 \\
& 1 brca & 10 \\
& 2 brca & 5 \\
Brca $<50$ & $\geqslant 3$ brca & 4 \\
& 1 brca & 7 \\
Brca $\geqslant 50$ & 2 brca & 4 \\
& $\geqslant 3$ brca & 10 \\
Ovca & 1 brca & 4 \\
& 2 brca & 2 \\
& $3 \geqslant$ brca & 2 \\
& 0 ovca & 1 \\
& 1 ovca & 1 \\
& $\geqslant 3$ ovca & 1 \\
\hline
\end{tabular}

`This woman had both breast and ovarian cancer. pared from the PBLs (isolated by ficoll-hypac centrifugation within 24 hours of phlebotomy) of $10 \mathrm{ml}$ heparin treated blood using Triazol (Gibco, Basel). cDNA was made using $10 \mu \mathrm{g}$ of total RNA, $500 \mathrm{ng}$ random hexamer, and 400 units of M-MLV reverse transcriptase (Gibco, Basel) in a $50 \mu \mathrm{l}$ volume according to the manufacturer's instructions.

PCR AND SEQUENCING

PCR for BRCA1 was performed as described previously. ${ }^{11}$ BRCA2 sequences were amplified in essentially the same manner, except that seven overlapping fragments of the BRCA2 coding sequence were amplified from either genomic DNA or cDNA as shown in fig 1 and table 1 . Segments 3 and 4 were PCR amplified from $200 \mathrm{ng}$ of genomic DNA template in a 25 $\mu \mathrm{l}$ reaction volume using standard conditions except that primer concentration was 200 $\mathrm{nmol} / 1$. The samples were cycled at $94^{\circ} \mathrm{C}$ for 30 seconds, $55^{\circ} \mathrm{C}$ for 30 seconds, $72^{\circ} \mathrm{C}$ for two minutes, 35 times. Segments $1,2,4$, and 5 were amplified from cDNA. A total of $0.5 \mu \mathrm{l}$ of cDNA was used in a nested PCR procedure in which the external primers were included for 20 cycles of amplification in a $10 \mu$ reaction volume. Then a $1 \mu \mathrm{l}$ aliquot was removed and used as template for an additional 30 cycles of amplification in a $25 \mu \mathrm{l}$ reaction in the presence of the internal primers. The temperature and time parameters were as above. Oligonucleotide primers were purchased from Microsynth (Balgach, Switzerland). Dideoxy sequencing of the PCR products was performed using Thermosequenase (Amersham, Buckinghamshire, UK) and analysed on a Licor automated sequencer (Lincoln, Nebraska).

PROTEIN TRUNCATION TEST

Transcription and translation was performed on $1 \mu \mathrm{l}$ of PCR product in a $5 \mu \mathrm{l}$ reaction using the TNT T7 coupled reticulocyte lysate system (Promega, Madison, WI). A total of $0.2 \mu \mathrm{l}$ of EasyTag ${ }^{35} \mathrm{~S}$ methionine (NEN, Boston, Mass) was included in each reaction. Labelled protein products were size separated using $12 \%$ sodium dodecyl sulphate polyacrylamide gel electrophoresis. Thereafter, the gels were fixed, dried, and exposed to Biomax film (Kodak, Rochester, NY).

\section{Results}

In this study 86 women were analysed using the protein truncation test for mutations in both BRCA1 and BRCA2. The results of BRCA1 
Table 3 BRCA1 and BRCA2 mutations and family characteristics

\begin{tabular}{lllllllll}
\hline Family & Gene & Exon & Nucl change & aa change & Type & Brca (age)* & Ovca (age)* & Other \\
\hline 1686 & BRCA1 & 11 & 1136 insA & 345 ter & F & - & $5(43-70)$ & - \\
$1604 \dagger$ & BRCA1 & 11 & 1611 delC & 502 ter & F & $5(41-42)$ & - & - \\
$1560 \dagger$ & BRCA1 & 11 & 1648 C $\rightarrow$ G & Ser510Stop & N & $5(33-42)$ & - & - \\
1564 & BRCA1 & 11 & 2804delAA & 901 ter & F & $3(33-59)$ & $2(37,64)$ & - \\
1668 & BRCA1 & 11 & 3039delTT & 990 ter & F & $4(40-65)$ & $2(?, ?)$ & Liver \\
$1540 \dagger$ & BRCA1 & 11 & 3449insA & 1114 ter & F & $4(39-48)$ & - & - \\
0323 & BRCA2 & 11 & 3036delACAA & 958 ter & F & $4(31-60)$ & - & Testis \\
1649 & BRCA2 & 11 & 3036delACAA & 958 ter & F & $3(35) \ddagger$ & - & - \\
1353 & BRCA2 & 14 & 7297delCT & 2358ter & F & $1(32)$ & - & 2 mel \\
\hline
\end{tabular}

$\mathrm{N}=$ nonsense mutation, $\mathrm{F}=$ frameshift mutation, del=deletion, ins=insertion, mel=melanoma.

*When more than two patients affected, age is given as a range.

$\dagger$ Reported previously. ${ }^{11}$

$\ddagger$ Age of the two other relatives unknown.

testing on 63 women have previously been reported but were included in this study since BRCA2 analysis had not been performed. A summary of their family histories is shown in table 2 .

Both cDNA and genomic DNA were used for mutation detection studies. All samples showing premature termination codons were verified by direct sequencing of genomic DNA. Two mutations were identified in cDNA and the remaining in genomic DNA. A summary of all mutations identified is shown in table 3.

BRCA1 analysis of the 23 women not included in our previous study ${ }^{11}$ showed three additional mutations which were spread throughout exon 11 of BRCA1. The mutation identified in family 1668 was a 2 base pair (bp) deletion at bp 3037 which resulted in a premature stop at bp 3091. The family history is remarkable in that there are two early onset ovarian cancer patients, five early onset breast cancer patients, and one 50 year old liver cancer patient who was unavailable for testing. In addition, two unaffected carriers were identified, one male (aged 45 years), the other female (aged 42). Affected persons in family 1564 harboured a 2 bp deletion at position 2804 which results in a premature stop codon $18 \mathrm{bp}$ later. This mutation has been reported previously but not published. Affected family members all presented with breast cancer under the age of 60 years; however, two of them developed ovarian cancer at the ages of 37 and 64 years. As with the previous family, two unaffected carriers were identified, one male (aged 27), the other female (aged 20). One ovarian cancer family without breast cancer was also included in this study. The mutation identified in this family (1686) was an A insertion at position 1129 of BRCA1 which resulted in a stop codon $17 \mathrm{bp}$ downstream and has not

Table 4 Haplotype sharing at the BRCA2 locus in families 0323 and 1649

\begin{tabular}{|c|c|c|c|c|c|c|c|}
\hline \multirow[b]{2}{*}{ Markers } & \multicolumn{6}{|c|}{ Family 0323} & \multirow{2}{*}{$\begin{array}{l}\text { Family } 1649 \\
7\end{array}$} \\
\hline & 1 & 2 & 3 & 4 & 5 & 6 & \\
\hline $\begin{array}{l}\text { D13S289 } \\
\text { D13S290 } \\
\text { D13S260 } \\
\text { D13S171 } \\
\text { D13S267 }\end{array}$ & $\begin{array}{ll}1 & 2 \\
1 & 4 \\
2 & 3 \\
2 & 5 \\
3 & 4\end{array}$ & $\begin{array}{ll}1 & 2 \\
1 & 4 \\
2 & 3 \\
2 & 5 \\
3 & 4\end{array}$ & $\begin{array}{ll}4 & 4 \\
3 & 1 \\
1 & 2 \\
2 & 2 \\
1 & 4\end{array}$ & $\begin{array}{l}1 \frac{4}{1} \\
\frac{1}{3} \\
\frac{1}{1} \frac{3}{4} \\
4 \frac{1}{1}\end{array}$ & $\begin{array}{ll}1 & 4 \\
3 & 3 \\
6 & 6 \\
4 & 4 \\
1 & 1\end{array}$ & $\begin{array}{l}1 \frac{4}{2} \\
2 \frac{1}{3} \\
\frac{1}{1} \frac{3}{4} \\
1 \frac{1}{1}\end{array}$ & $\begin{array}{l}2 \frac{4}{1} \\
\frac{1}{3} \\
\frac{1}{2} \frac{3}{4} \\
4 \frac{1}{1}\end{array}$ \\
\hline
\end{tabular}

Patients $1-6$ belong to family 0323 as indicated in fig $2 A$ and patient 7 belongs to family 1649 (fig 2C). Common alleles in persons harbouring the delACAA mutation (underlined) can be seen within family 0323 which are present in patient 7 (family 1649). Segregating alleles for marker D13S260 could not be determined, so both alleles are underlined. been reported previously. All patients within this family presented with ovarian cancer under the age of 70 years, the youngest of whom was only 43 years of age when diagnosed.

Since 80 of the selected families meeting our inclusion requirements did not appear to harbour mutations in BRCA1, BRCA2 screening was performed. Three families were identified as having mutations in BRCA2. Affected persons in family 0323 harbour a $4 \mathrm{bp}$ deletion (ACAA) at position 3036 which results in a stop codon $67 \mathrm{bp}$ downstream.

Three patients with early onset breast cancer harboured the $4 \mathrm{bp}$ deletion and an obligate carrier was identified who died of ovarian cancer three years after her diagnosis at 66 years of age. In addition, a 60 year old sister of the ovarian cancer patient was diagnosed with breast cancer but did not harbour the mutation nor did her 40 year old son who suffered from testicular carcinoma (fig 2A). Family 1649 (fig 2B) harboured an identical mutation to that found in family 0323 which was associated only with early onset breast cancer. In this family, three generations of women developed early onset breast cancer. The paternal grandmother of the proband also developed breast cancer albeit at a later age, but she was unavailable for testing. Haplotype analysis showed that these two families are related, as common alleles were shared between affected persons (table 4 ). The mutation found in families 0323 and 1649 is similar to that reported previously ${ }^{11}{ }^{12}$ in that it occurred in a string of adenine bases. The exact location of the mutation, however, is impossible to determine since the surrounding sequence (GTGATAAACAAGCAA) does not allow the differentiation between a deletion starting at position 3034,3035 , or 3036 .

A $2 \mathrm{bp}$ deletion at position 1297 in exon 14 of BRCA2 resulting in a premature stop codon $3 \mathrm{bp}$ downstream of the mutation was identified in the index patient from family 1353 and is a new mutation. This woman was the only breast cancer patient in the family (fig 2C). Additional family members suffered from other malignancies which included two melanoma patients, one of whom developed disease at 30 years of age, and one basal cell carcinoma patient. Unfortunately, none of these patients was available for analysis. 
A 0323
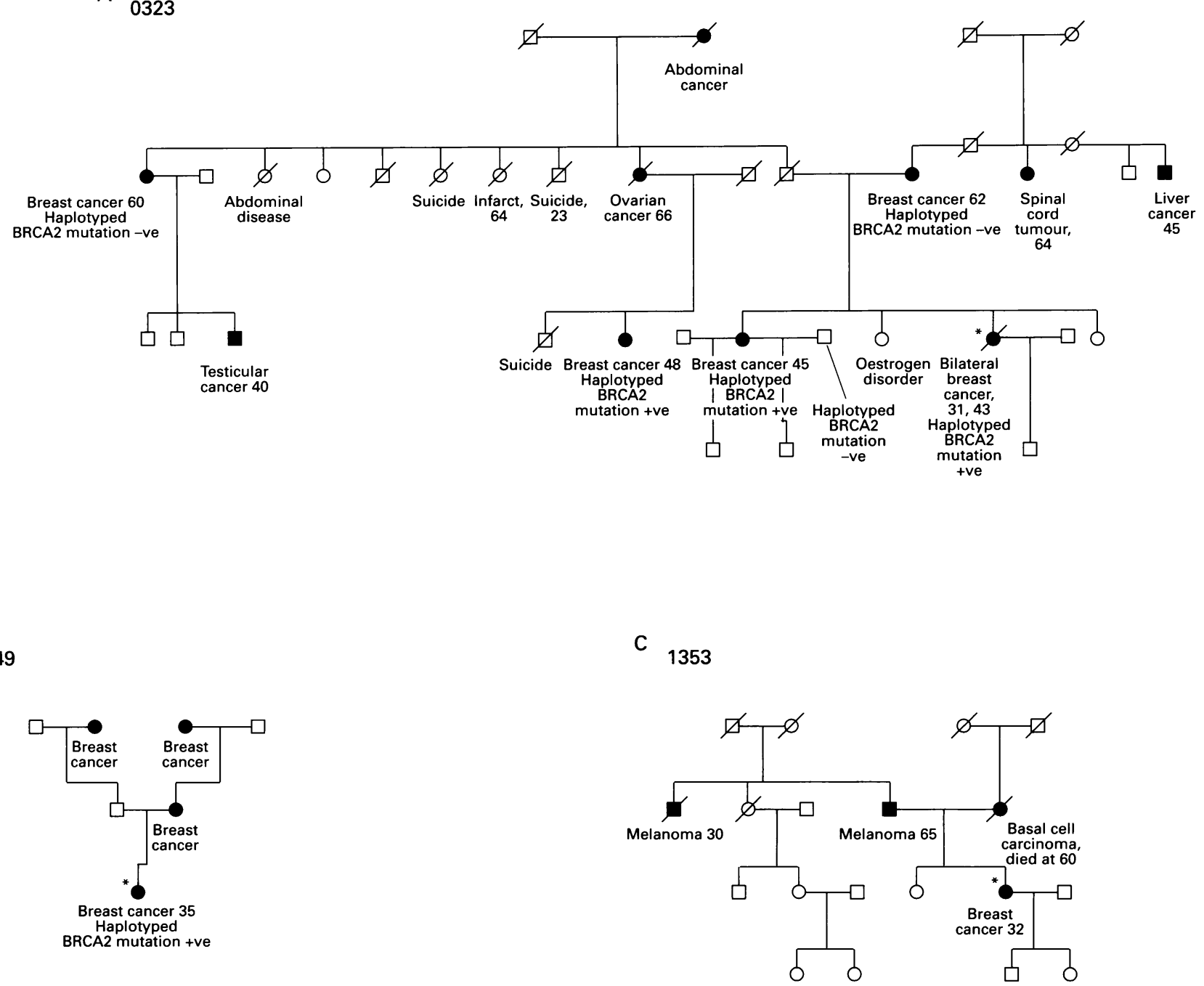

Figure 2 The three pedigrees with BRCA2 mutations. Persons used for haplotyping analysis are as indicated. Those persons with an asterisk above their symbol are the index patients from each of the families and have the same BRCA2 mutation (indicated as BRCA2 +ve). Age of diagnosis is indicated where known.

\section{Discussion}

Most studies on breast cancer families have focused on large informative families where there is reasonably good pedigree data. Relatively little is known about persons who have minimal family history of disease but have developed disease at an unusually early age. In this study we have addressed this issue by including in the study persons who fulfil a minimal criteria. The carrier frequency for BRCA1 is $1: 833^{13}$ and BRCA2 is approximately the same, ${ }^{6}$ suggesting 1:400 people in the general population are carriers. The two genes are expected to account for approximately $10 \%$ of the breast cancer cases under 45 years of age. ${ }^{14}$ Therefore it is to be expected that approximately half of the mutations would be the result of BRCA1 and half BRCA2. In this study the ratio of BRCA1 to BRCA2 mutations was $3: 1$, indicating that BRCAl is more frequent in the Swiss population than BRCA2. Since, however, only a few mutations were detected, this result may be inaccurate owing to sample size.

Because of the size of BRCA1 and BRCA2, efficient screening of both genes remains problematical. The PTT represents a relatively useful alternative to more labour intensive screen- ing strategies in that large amounts of coding sequence can be quite rapidly examined. A major advantage of this method, in comparison to other detection strategies, is that any changes observed are likely to lead to a loss of function. In this report cDNA was used as template for the PTT which is generated from mRNA by reverse transcriptase. Mutant message may be susceptible to the actions of nonsense mediated decay and therefore may not be represented to the same extent as normal message ${ }^{15}$ which would result in the apparent absence of detectable truncated products by the PTT. This, we believe, is unlikely since before starting this study we investigated lymphoblastoid cell lines with known mutations that had previously been reported as being susceptible to the actions of nonsense mediated decay. These studies indicated that as long as mRNA was isolated from lymphocytes immediately after phlebotomy, sufficient mutant message was present for $\mathrm{CDNA}$ conversion and hence mutation detection (results not shown). An additional feature of the PTT which could lead to decreased sensitivity of the method is the amount of overlap between the respective segments used for gene analysis. If insufficient overlap is designed into the 
method, a loss of sensitivity is expected. In this study fragment overlap was approximately 200 bp which is sufficient to ensure that mutations occurring towards the ends of each segment would be detected. There remain, however, limitations with respect to the sensitivity of the PTT. It does not detect missense mutations which have been shown to account for a small but significant proportion of changes in BRCA1 and BRCA2. Preliminary results from a second screening of 23 women included in this study for additional BRCA1 mutations showed five polymorphisms of unknown significance (data not shown), suggesting that the PTT should not be relied on as a definitive test. Thus the PTT can be recommended for an initial screen for both BRCA1 and BRCA2; however, other more sensitive screening strategies such as denaturing gradient gel electrophoresis should also be used. ${ }^{16}$ Alternatively, indirect mutation detection methods could be used, such as microsatellite marker analysis or restriction fragment length polymorphism (RFLP) analysis which could indicate the disease allele in cases where there were genomic deletions, rearrangements, promotor sequence mutations, and missense mutations.

Out of 86 breast cancer patients who fulfilled our inclusion criteria, only $10.5 \%$ were found to harbour mutations in either BRCA1 or 2 . Interestingly, all persons with either BRCA1 or 2 mutations had a significant history of cancer; however, it appears that BRCA2 mutations may be less tissue specific than BRCA1 mutations.

If age restrictions are applied, then the contribution of an inherited susceptibility to the overall number of breast cancer patients should increase as age decreases. ${ }^{17}$ In the families studied in this report only about $10 \%$ could be assigned to either BRCA1 or 2 which is close to the predicted value. ${ }^{17}$ These results are comparable to recently published data indicating that BRCA1 and BRCA2 do not account for all breast/ovarian cancer families. ${ }^{16}{ }^{18}$ Given the selection criteria adopted for the present study the mutation detection rate of $10.5 \%$ is consistent with other larger studies using slightly different inclusion requirements ${ }^{1618}$ and suggests that the Swiss population is similar to others from the same region. The paucity of genetic changes found in women who did not have a family history of disease supports the notion that BRCA1 and BRCA2 are unlikely to be associated with de novo mutations and therefore represent a special entity with respect to breast cancer development.

Since BRCA1 has been linked to both breast and ovarian cancer it was not surprising to identify a germline BRCA1 mutation in an ovarian cancer family. The mutation occurs in the $5^{\prime}$ end of the gene, thus providing further evidence that mutations towards the 5 ' end appear to be associated with an increased ovarian cancer risk. ${ }^{19}$ BRCA2 carriers, however, do not have the same risk of ovarian cancer development but do nevertheless have a greater risk than the general population. Recently, it has been shown that there is an ovarian cancer susceptibility region in BRCA $2,{ }^{7}$ somewhere between nucleotide 4235 and 6504 , known as the ovarian cancer cluster region (OCCR). The BRCA2 family with ovarian cancer identified here harbours a mutation at 3036 which has previously been reported ${ }^{12}$ to be associated with ovarian cancer and may therefore extend the OCCR in BRCA2 by another $1200 \mathrm{bp}$ towards the 5 ' end.

The spectrum of disease observed in families harbouring BRCA2 mutations was different from that associated with mutations in BRCA1. It appears that mutations in BRCA2 may confer a broader range of disease susceptibility as compared to BRCA1. Indeed it has been shown that germline mutations in BRCA2 appear to be associated with pancreatic cancer development. ${ }^{21}$ In the current study we observed two melanoma patients within one family where only one isolated early onset breast cancer case was observed, suggesting that BRCA2 may confer increased risks for other types of cancer than breast and ovary. Unfortunately, DNA was not available for study from these two patients.

Two families from different regions of Switzerland were identified harbouring the same germline BRCA2 mutation (delACAA). As this $4 \mathrm{bp}$ deletion is not discernible, owing to the nature of the surrounding sequence, from those reported at positions 3034 or 3035 , it is difficult to determine if this region represents a common site for mutation or a founder mutation. Haplotyping analysis of the two families reported here suggests that these two families are related as common alleles are shared by all affected persons. Since this mutation has been previously described in families from different countries it remains possible that it is either a founder mutation or is a regional hotspot for mutations.

The remaining 77 patients in whom germline BRCA1 or BRCA2 mutations were not identified represent a significant problem with respect to mutation detection and genetic counselling. Given that only a few families tested positive, it is to be expected that some mutations were not detected using the PTT, however, it is unlikely that a significant number have been missed, implying that there are other genes responsible for the increased risk of breast cancer development seen in this population of women.

This work was supported in part by grants AKT332, AKT463 from the Swiss Cancer League, the Krebsliga Beider Basel, The Roche Research Foundation, the Ciba-Geigy Jubilaums Stiftung and the Freiwillige Medizinische Akademische Gesellschaft.

1 Miki Y, Swensen J, Shattuck-Eidens D, et al. A strong candidate for the breast and ovarian cancer susceptibility gene date for the breast and ovarian 1 .

2 Wooster R, Bignell G, Lancaster J, et al. Identification of the breast cancer susceptibility gene BRCA2. Nature 1995;378: 789-92.

3 Hall JM, Lee MK, Newman B, et al. Linkage of early-onse familial breast cancer to chromosome 17q21. Science 1990 250:1684-49.

4 Wooster R, Neuhausen SL, Mangion J, et al. Localization of breast cancer susceptibility gene BRCA2, to chromosome 13q12-13. Science 1994;265:2088-90. 
5 Easton DF, Ford D, Bishop DT, Consortium BC. Breas and ovarian cancer incidence in BRCA1-mutation carriers. Am $\mathcal{F}$ Hum Genet 1995;56:265-71.

6 Ford D, Easton DF. The genetics of breast and ovarian cancer. Br f Cancer 1995;72:805-12.

7 Gayther SA, Mangion J, Russel P, et al. Variation of risks of breast and ovarian cancer associated with differen germline mutations of the BRCA2 gene. Nat Gene 1997;15:103-5.

8 Wu LC, Wang ZW, Tsan JT, et al. Identification of a RING protein that can interact in vivo with BRCA1 gene product. Nat Genet 1996;14:430-40.

9 Shattuck-Eidens D, McClure M, Simard J, et al. A collaborative survey of 80 mutations in the BRCA1 breast and ovarian cancer susceptibility gene: implications for presymptomatic testing and screening. $\mathcal{F} A M A$ 1995;273:535-

10 Miller SA, Dykes DD, Polesky HF. A simple salting out procedure for extracting DNA from human nucleated cells. Nucleic Acids Res 1988;53:739.

11 Garvin AM, Spycher M, Haner H, et al. BRCA1 mutations in a selected series of breast/ovarian cancer patients. $\mathcal{f} \mathrm{Med}$ Genet 1996;33:721-5.

12 Lenoir et al, 1996. Breast Cancer Information Core http:// www.nchgr.nih.gov/Intramural_research/Lab_transfer/Bic/

13 Takahashi H, Chiu HC, Bandera CA, et al. Mutations of the BRCA2 gene in ovarian carcinomas. Cancer Res 1996;56: 2738-41.

14 Ford D, Easton DF, Peto J. Estimates of the gene frequency of BRCA1 and its contribution to breast and ovarian cancer incidence. Am $\mathcal{F}$ Hum Genet 1995;57:1457-62.
15 Fitzgerald MG, Macdonald DJ, Krainer M, et al. Germline BRCA1 mutations in jewish and non-jewish women with early-onset breast cancer. $N$ Engl f Med 1996;334:143-9.

16 Stoppa-Lyonnet D, Laurent-Puig P, Essioux L, et al. BRCAl sequence variations in 160 individuals referred to a breast/ovarian family cancer clinic. Am $\mathcal{f}$ Hum Genet 1997;60:1021-30.

17 Friedman LS, Szabo CL, Ostermeyer EA, et al. Novel inherited mutations and variable expressivity of BRCA1 alleles, including founder mutations 185 delAG in Ashkenazi Jewish families. Am f Hum Genet 1995;57:1284Ashk

18 Hakansson S, Johannsson O, Johansson U, et al. Moderate frequency of BRCA1 and BRCA2 germ-line mutations in Scandinavian familial breast cancer. Am $\mathcal{f}$ Hum Genet 1997;60:1068-78.

19 Langston AA, Malone KE, Thompson JD, Daling JR Ostrander EA. BRCA1 mutations in a population-based
sample of young women with breast cancer. $N$ Engl $\mathcal{F} M e d$ sample of young wom

20 Gayther SA, Warren W, Mazoyer S, et al. Germline mutations of the BRCAl gene in breast and ovarian families provide evidence for a genotype-phenotype correlation. Nat Genet 1996;11:428-33.

21 Goggins M, Schutte M, Lu J, et al. Germline BRCA2 gene mutations in patients with apparently sporadic pancreatic carcinomas. Cancer Res 1996;56:5360-4.

22 Tavtigian S, Simard J, Rommens J, et al. The complete BRCA2 gene and mutations in chromosome 13q-linked kindreds. Nat Genet 1996;12:333-7. 